\title{
Lifting matroid divisors on tropical curves
}

\section{Dustin Cartwright}

*Correspondence: cartwright@utk.edu Department of Mathematics, University of Tennessee, 227 Ayres Hall, Knoxville, TN 37996-1320, USA

\begin{abstract}
Tropical geometry gives a bound on the ranks of divisors on curves in terms of the combinatorics of the dual graph of a degeneration. We show that for a family of examples, curves realizing this bound might only exist over certain characteristics or over certain fields of definition. Our examples also apply to the theory of metrized complexes and weighted graphs. These examples arise by relating the lifting problem to matroid realizability. We also give a proof of Mnëv universality with explicit bounds on the size of the matroid, which may be of independent interest.
\end{abstract}

Keywords: Tropical curve, Matroid, Lifting, Specialization inequality

Mathematics Subject Classification: Primary 14T05, 05B35

\section{Background}

The specialization inequality in tropical geometry gives an upper bound for the rank of a divisor on a curve in terms of a combinatorial quantity known as the rank of the specialization of the divisor on the dual graph of the special fiber of a degeneration [6]. This bound can be sharpened by incorporating additional information about the components of the special fiber, giving augmented graphs [2] or metrized complexes [1]. All of these inequalities can be strict because there may be many algebraic curves and divisors with the same specialization. Thus, the natural question is whether, for a given graph and divisor on that graph, the inequality is sharp for some algebraic curve and divisor. If $R$ is the discrete valuation ring over which the degeneration of the curve is defined, we will refer to such a curve and divisor as a lifting of the graph with its divisor over $R$. In this paper, we show that the existence of a lifting can depend strongly on the characteristic of the field:

Theorem 1.1 Let Pbeany finite set of prime numbers. Then there exist graphs $\Gamma$ and $\Gamma^{\prime}$ with rank 2 divisors $D$ on $\Gamma$ and $D^{\prime}$ on $\Gamma^{\prime}$ with the following property: For any infinite field $k, \Gamma$ and $D$ lift over $k[[t]]$ if and only if the characteristic of $k$ is in $P$, and $\Gamma^{\prime}$ and $D^{\prime}$ lift over $k[t]]$ if and only if the characteristic of $k$ is not in $P$.

We also show that the existence of a lift depends on the field even beyond characteristic:

C 2015 Cartwright. This article is distributed under the terms of the Creative Commons Attribution 4.0 International License (http:// creativecommons.org/licenses/by/4.0/), which permits unrestricted use, distribution, and reproduction in any medium, provided you give appropriate credit to the original author(s) and the source, provide a link to the Creative Commons license, and indicate if changes were made. 
Theorem 1.2 Let $k^{\prime}$ be any number field. Then there exists a graph $\Gamma$ with a rank 2 divisor $D$ such that for any field $k$ of characteristic $0, \Gamma$ and $D$ lift over $k[[t]]$ if and only if $k$ contains $k^{\prime}$.

Both Theorem 1.1 and 1.2 are immediate consequences of the following:

Theorem 1.3 Let $X$ be a scheme of finite type over Spec $\mathbb{Z}$. Then there exists a graph $\Gamma$ with a rank 2 divisor $D$ such that, for any infinite field $k$, $\Gamma$ and $D$ lift overk[[t]] if and only if $X$ has a k-point.

Theorems 1.1, 1.2, and 1.3 all apply equally well to divisors on weighted graphs [2] because the construction of a degeneration in Theorem 1.3 uses curves of genus 0 in the special fiber and for such components, the theory of weighted graphs agrees with unweighted graphs.

Moreover, these theorems also apply to the metrized complexes introduced in [1], which record the isomorphism types of the curves in the special fiber. Again, for rational components, the rank of the metrized complex will be the same as the rank for the underlying graph. For metrized complexes, there is a more refined notion of a limit $g_{d}^{r}$, which involves additionally specifying vector spaces of rational functions at each vertex. Not every divisor of degree $d$ and rank $r$ on a metrized complex lifts to a limit $g_{d}^{r}$, but the examples from the above theorems do:

Proposition 1.4 Let $\Gamma$ and $D$ be a graph and divisor constructed as in Theorem 1.3. Then for any lift of $\Gamma$ to a metrized complex with rational components, there also exists a lift of $D$ to a limit $g_{d}^{2}$.

If we were to consider divisors of rank 1 rather than rank 2, [3] provides a general theory for lifting. They prove that if a rank 1 divisor can be lifted to a tame harmonic morphism with target a genus 0 metrized complex, then it lifts to a rank 1 divisor an algebraic curve. Moreover, the converse is true except for possibly some cases of wild ramification in positive characteristic. Using this, they give examples of rank 1 divisors which do not lift over any discrete valuation ring [3, Sec. 5]. While the existence of a tame harmonic morphism depends on the characteristic, the dependence is only when the characteristic is at most the degree of the divisor [3, Rmk. 3.9]. In contrast, lifting rank 2 divisors can depend on the characteristic even when the characteristic is bigger than the degree:

Theorem 1.5 If $P=\{p\}$ where $p \geq 443$ is prime, then the divisors $D$ and $D^{\prime}$ in Theorem 1.1 can be taken to have degree less than $p$.

For simplicity, we have stated Theorems 1.1, 1.2, and 1.3 in terms of liftings over rings of formal power series, but some of our results also apply to other discrete valuation rings. In particular, these theorems apply verbatim with $k[[t]]$ replaced by any DVR which contains its residue field $k$. For other, possibly even mixed characteristic DVRs, we have separate necessary and sufficient conditions in Theorems 3.4 and 3.5, respectively. 
The proof of Theorem 1.3 and its consequences use Mnëv's universality theorem for matroids [20]. Matroids are combinatorial abstractions of vector configurations in linear algebra. However, not all matroids come from vector configurations and those that do are called realizable. Mnëv proved that realizability problems for rank 3 matroids in characteristic 0 can encode arbitrary systems of integral polynomial equations and Lafforgue extended this to arbitrary characteristic [17, Thm. 1.14]. Thus, Theorem 1.3 follows from universality for matroids together with a connection between matroid realizability and lifting problems, which is done in Theorems 3.4 and 3.5. We also give a proof of universality in arbitrary characteristic with explicit bounds on the size of the matroid to establish Theorem 1.5.

Matroids have appeared before in tropical geometry and especially as obstructions for lifting. For example, matroids yield examples of matrices whose Kapranov rank exceeds their tropical rank, showing that the minors do not form a tropical basis [11, Sec. 7]. In addition, Ardila and Klivans defined the tropical linear space for any simple matroid, which generalizes the tropicalization of a linear space [5]. The tropical linear spaces are realizable as the tropicalization of an algebraic variety if and only if the matroid is realizable [16, Cor. 1.5]. This paper is only concerned with rank 3 matroids, which correspond to two-dimensional fans and the graphs for which we construct lifting obstructions are the links of the fine subdivision of the tropical linear space (the fine subdivision is defined in [5, Sec. 3]).

Moreover, the matroid divisors from this paper have found applications to other questions regarding the divisor theory of graphs. David Jensen has shown that the matroid divisor of the Fano matroid gives an example of a 2-connected graph which is not BrillNoether general for any metric parameters [14]. In addition to the Baker-Norine rank of a divisor used in this paper, Caporaso has given a definition of the algebraic rank of a divisor, which involves quantifying over all curves over a given field [9]. Yoav Len has shown that in contrast to the results in Sect. 2, the algebraic rank of a matroid divisor detects realizability of the matroid, and he has used this to show that the algebraic rank can depend on the field [19].

Since rank 3 matroids give obstructions to lifting rank 2 divisors on graphs, it is natural to wonder if higher rank matroids give similar examples for lifting higher rank divisors. While we certainly expect there to be results similar to Theorems 1.1, 1.2, and 1.5 for divisors on graphs which have ranks greater than 2, it is not clear that higher rank matroids would provide such examples, or even what the right encoding of the matroid in a graph would be. From a combinatorial perspective, our graphs are just order complexes of the lattice of flats, but for higher rank matroids, the order complex is a simplicial complex but not a graph.

This paper is organized as follows. In Sect. 2, we introduce the matroid divisors which are our key class of examples and show that as combinatorial objects they behave as if they should have rank 2. In Sect. 3, we relate the lifting of matroid divisors to the realizability of the matroid. Section 4 looks at the applicability of our matroid to the question of lifting tropically Brill-Noether general divisors and shows that, with a few exceptions, matroid divisors are not Brill-Noether general. Finally, Sect. 5 provides a quantitative proof of Mnëv universality as the basis for Theorem 1.5. 


\section{Matroid divisors}

In this section, we construct the divisors and graphs that are used in Theorem 1.3. As in [6] and [7], we will refer to a finite formal sum of the vertices of a graph as a divisor on that graph. Divisors are related by so-called "chip-firing moves" in which the weight at one vertex is decreased by its degree and those of its neighbors are correspondingly each increased by 1 . A reverse chip-firing move is the inverse operation.

As explained in the Sect. 1, the starting point in our construction is a rank 3 simple matroid. A matroid is a combinatorial model for an arrangement of vectors, called elements, in a vector space. A rank 3 simple matroid corresponds to such an arrangement in a three-dimensional vector space, for which no two vectors are multiples of each other. There are many equivalent descriptions of a matroid, but we will work with the flats, which correspond to vector spaces spanned by subsets of the arrangement, and are identified with the set of vectors that they contain. For a rank 3 simple matroid, there is only one rank 0 and one rank 3 flat, and the rank 1 flats correspond to the elements of the matroid, so our primary interest will be in rank 2 flats. Throughout this paper, flat will always refer to a rank 2 flat.

We refer the reader to [21] for a thorough reference on matroid theory, or [15] for an introduction aimed at algebraic geometers. However, in the case of interest for this paper, we can give the following axiomatization:

Definition 2.1 A rank 3 simple matroid $M$ consists of a finite set $E$ of elements and a collection $F$ of subsets of $E$, called the flats of $M$, such that any pair of elements is contained in exactly one flat, and such that there are at least two flats. A basis of such a matroid is a triple of elements which are not all contained in a single flat.

By projectivizing the vector configurations above, a configuration of distinct $k$-points in the projective plane $\mathbb{P}_{k}^{2}$ determines a matroid. The elements of this matroid are the points of the configuration and the flats correspond to lines in $\mathbb{P}_{k}^{2}$, identified with the points contained in them. A matroid coming from a point configuration in this way is called realizable over $k$ and in Sect. 3, we will use the fact that matroid realizability can depend on the field.

Given a rank 3 simple matroid $M$ with elements $E$ and flats $F$, we let $\Gamma_{M}$ be the bipartite graph with vertex set $E \amalg F$, and an edge between $e \in E$ and $f \in F$ when $e$ is contained in $f$. The graph $\Gamma_{M}$ is sometimes called the Levi graph of $M$. We let $D_{M}$ be the divisor on the graph $\Gamma_{M}$ consisting of the sum of all vertices corresponding to elements of the ground set $E$.

Proposition 2.2 The divisor $D_{M}$ has rank 2 .

Proof To prove the proposition, we first need to show that for any degree 2 effective divisor $E$, the difference $D_{M}-E$ is linearly equivalent to an effective divisor. We build up a "toolkit" of divisors linearly equivalent to $D_{M}$. First, for any flat $f$, we can reverse fire $f$. This moves a chip from each element contained in $f$ to $f$ itself. Thus, the result is an effective divisor whose multiplicity at $f$ is the cardinality of $f$, which is at least 2 . Our second chip-firing move is to reverse fire a vertex $e$ as well as all flats containing $e$. The net effect 
will be no change at $e$ but each neighbor $f$ of $e$ will end with $|f|-1 \geq 1$ chips. Third, we will use the second chip-firing move, after which all the flats which contain $e$ have at least one chip, after which it is possible to reverse fire $e$ again.

Now let $E$ be any effective degree 2 divisor on $\Gamma_{M}$. Thus, $E$ is the sum of two vertices of $\Gamma_{M}$. We consider the various combinations which are possible for these vertices. First, if $E=[e]+\left[e^{\prime}\right]$ for distinct elements $e$ and $e^{\prime}$, then $\Gamma_{M}-E$ is effective. Second, if $E=[e]+[f]$, then we have two subcases. If $e$ is in $f$, then we reverse fire $e$ and all flats containing it. If $e$ is not in $f$, then we can reverse fire just $f$. Third, if $E=[f]+\left[f^{\prime}\right]$ for distinct flats $f$ and $f^{\prime}$, then there are again two subcases. If $f$ and $f^{\prime}$ have no elements in common, then we can reverse fire $f$ and $f^{\prime}$. If $f$ and $f^{\prime}$ have a common element, say $e$, then we reverse fire $e$ together with the flats which contain it. Fourth, if $E=2[e]$, then we use the third chip-firing move, which will move one chip onto $e$ for each flat containing $e$, of which there are at least 2. Fifth, if $E=2[f]$, then we reverse fire $f$.

Finally, to show that the rank is at most 2, we give an effective degree 3 divisor $E$ such that $D_{M}-E$ is not linearly equivalent to any effective divisor. For this, let $e_{1}, e_{2}$, and $e_{3}$ form a basis for $M$ and let $f_{i j}$ be the unique flat containing $e_{i}$ and $e_{j}$ for $1 \leq i<j \leq 3$. We set $E=\left[f_{12}\right]+\left[f_{13}\right]+\left[f_{23}\right]$ and claim that $D_{M}-E$ is not linearly equivalent to any effective divisor. We reverse fire $e_{1}$ together with all flats containing it to get the following divisor linearly equivalent to $D_{M}-E$ :

$$
\left[e_{1}\right]+\left(\left|f_{12}\right|-2\right)\left[f_{12}\right]+\left(\left|f_{13}\right|-2\right)\left[f_{13}\right]-\left[f_{23}\right]+\sum_{\substack{f_{k} \ni e_{1} \\ f_{k} \neq f_{12} f_{13}}}\left(\left|f_{k}\right|-1\right)\left[f_{k}\right]
$$

which is effective except at $f_{23}$.

We wish to show the divisor in (1) is not linearly equivalent to any effective divisor, which we will do by showing that it is $f_{23}$-reduced using Dhar's burning algorithm [12]. We first claim that for any element $e$ other than $e_{1}$, there is a path from $f_{23}$ to $e$ which does not encounter any chips. If $e$ is in $f_{23}$, then there is a direct edge between these vertices. If not, then we first let $f$ denote the unique flat containing both $e$ and $e_{1}$. Since $e_{1}, e_{2}$, and $e_{3}$ form a basis, they cannot all be contained in $f$. Without loss of generality, we can assume that $e_{2}$ is not in $f$, and so $e, e_{1}$, and $e_{2}$ form a basis. Thus, if $f^{\prime}$ is the unique flat containing $e_{2}$ and $e$, then $f^{\prime}$ does not contain $e_{1}$. Therefore, the path from $f_{23}$ to $e_{2}$ to $f^{\prime}$ to $e$ does not cross any chips.

By the claim in the previous paragraph, the burning algorithm will lead to $\left|f_{12}\right|-1$ independent "fires" arriving at $f_{12}$, one for each element in $f_{12} \backslash e_{1}$. Thus, these remove the $\left|f_{12}\right|-2$ chips on $f_{12}$ and one path continues on to remove the single chip from $e_{1}$. Likewise, $\left|f_{13}\right|-1$ independent "fires" arrive at $\left|f_{13}\right|-2$, and one continues on and passes through $e_{1}$ to arrive at all the flats containing $e_{1}$. Therefore, for every flat $f$ that contains $e_{1}$, other than $f_{12}$ and $f_{13}$, which have already been handled, there is a "fire" arriving from every element of $f$, which exceeds the $|f|-1$ chips on this vertex. The paths used to cover $\Gamma_{M}$ are summarized schematically in Fig. 1. Since the burning algorithm covers the graph $\Gamma_{M}$, we conclude that the divisor $D_{M}-E$ is $f_{23}$-reduced and so not linearly equivalent to an effective divisor. 


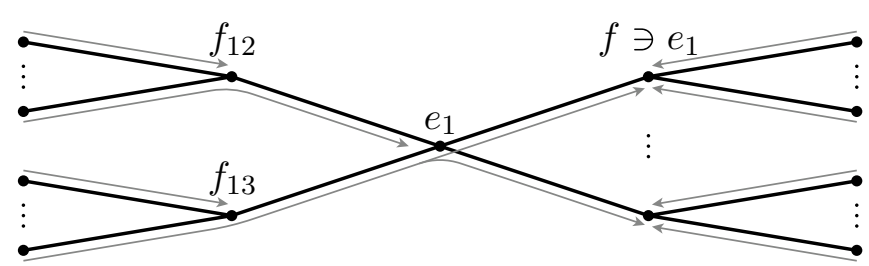

Fig. 1 Paths through the graph $\Gamma_{M}$ taken by the burning algorithm applied to show the divisor (1) is $f_{23}$ -reduced. There is a path from $f_{23}$ to any element other than $e_{1}$, which are depicted on the left and right, and from these vertices, there are paths which eventually equal or exceed the number of chips at all vertices of the graph

Proposition 2.2 also shows that if $\Gamma_{M}$ is made into a weighted graph by giving all vertices genus 0 , then $D_{M}$ has rank 2 on the weighted graph. The rank is, again, unchanged for any lifting of the weighted graph to a metrized complex. To show that $D_{M}$ is also a limit $g_{d}^{2}$ as in Proposition 1.4, we also need to choose three-dimensional vector spaces of rational functions on the variety attached to each vertex.

Proof of Proposition 1.4 We recall from [1] that a lift of $\Gamma_{M}$ to a metrized complex means associating a $\mathbb{P}_{k}^{1}$ for each vertex $v$ of the graph, which we denote $C_{v}$, and a point on $C_{v}$ for each edge incident to $v$. A lift of the divisor $D_{M}$ is a choice of a point on $C_{e}$ for each element $e$ of $M$.

The data of a limit $g_{d}^{2}$ is a three-dimensional vector space $H_{v}$ of rational functions on each $C_{v}$ [1, Sec. 5], which we choose as follows. For each flat $f$, we arbitrarily choose two elements from it and let $p_{f, 1}$ and $p_{f, 2}$ be the points on $C_{f}$ corresponding to the edges from $f$ to each of the chosen elements. Our vector space $H_{f}$ consists of the rational functions which have at worst simple poles at $p_{f, 1}$ and $p_{f, 2}$. For each element $e$, we choose an arbitrary flat containing $e$ and let $q_{e}$ be the point on $C_{e}$ corresponding to the edge to $e$. Our vector space $H_{e}$ consists of the rational functions which have at worst poles at $q_{e}$ and at the point of the lift of $D_{M}$.

Now to check that these vector spaces form a limit $g_{d}^{2}$, we need to show that the refined rank is 2. For this, we use the same "toolkit" functions as in the proof of Proposition 2.2, but we augment them with rational functions from the prescribed vector spaces on the algebraic curves. The first item from our toolkit was reverse firing a flat $f$ to produce at least two points on $C_{f}$. We can use rational functions with poles at $p_{f, 1}$ and $p_{f, 2}$ to produce any degree two effective divisor on $C_{f}$. For each $C_{e}$ such that $e$ is an element of $f$, we need to use a rational function with a zero at the edge to $f$ and a pole at the lift of the divisor $D_{M}$.

The second item we needed in our toolkit was reverse firing an element $e$ together with all of the flats which contain it. Here, for each element $e^{\prime}$ other than $e$, we use the rational function with a pole at the divisor and a zero at the point corresponding to the edge to the unique flat containing both $e^{\prime}$ and $e$. At each flat $f$ containing $e$, we can use any function with a pole at $p_{f, i}$, where $i \in\{1,2\}$ can be chosen to not be the edge leading to $e$. This produces a divisor at an arbitrary point of $C_{e}$.

The third and final operation we used was the previous item followed by a reverse firing of $e$. Here, we use the same rational functions as before, but we can choose any 
rational function on $C_{e}$ which has poles at the point of the divisor and $q_{e}$, thus giving us two arbitrary points on $C_{e}$. We conclude that rational functions can be found from the prescribed vector spaces to induce a linear equivalence between the lift of $D_{M}$ and any two points on the metrized complex.

In the case of rank 1 divisors, lifts can be constructed using the theory of harmonic maps of metrized complexes, which gives a complete theory for divisors defining tamely ramified maps to $\mathbb{P}^{1}[3]$. A sufficient condition for lifting a rank 1 divisor is for it to be the underlying graph of a metrized complex which has a tame harmonic morphism to a tree (see [4, Sec. 2] for precise definitions). These definitions are limited to the rank 1 case, but for rank 2 divisors we can subtract points to obtain a divisor of rank at least 1 . In particular, if $D_{M}$ lifts, then for any element $e, D_{M}-[e]$ will be the specialization of a rank 1 effective divisor. However, the lifting criterion of [3] is satisfied for these subtractions, independent of the liftability of $D_{M}$.

Proposition 2.3 Let $M$ be any rank 3 simple matroid and e any element of $M$. Also, let $k$ be an algebraically closed field of characteristic not 2. Then, $\Gamma_{M}$ has a tropical modification $\widetilde{\Gamma}_{M}$ such that $\widetilde{\Gamma}_{M}$ can be lifted to a totally degenerate metrized complex over $k$ with a tame harmonic morphism to a genus 0 metrized complex, such that one fiber is a lift of the divisor $D_{M}-[e]$.

Proof We first construct a modification $\widetilde{\Gamma}_{M}$ of $\Gamma_{M}$ which has a finite harmonic morphism from $\widetilde{\Gamma}_{M}$ to a tree $T$. The tree $T$ will be a star tree with a central vertex $w$, together with an unbounded edge, denoted $r_{f}$, for each flat $f$ which does not contain $e$, and a single unbounded edge $r_{e}$ corresponding to $e$. Our modification of $\Gamma_{M}$ consists of adding the following unbounded edges: at $e$, we add one unbounded edge $s_{e, f}$ for each flat $f$ containing $e$. At each element $e^{\prime}$ other than $e$, we add one unbounded edge $s_{e^{\prime}, f}$ for each flat $f$ which contains neither $e$ nor $e^{\prime}$. At a flat $f$, we add unbounded edges $s_{f, i}$ where $i$ ranges from 1 to $|f|$ if $e \notin f$ and from 1 to $|f|-2$ if $e \in f$.

We now construct a finite harmonic morphism $\phi$ from $\widetilde{\Gamma}_{M}$ to $T$. Each element other than $e$ maps to the central vertex $w$ of $T$. Each flat $f$ not containing $e$ maps to a point one unit of distance along the corresponding ray $r_{f}$ of $T$. Then the rays $s_{e^{\prime}, f}$ and $s_{f, i}$ also map to the ray $r_{f}$, starting at $w$ and $\phi(f)$, respectively.

We map the vertex $e$ to its unbounded ray $r_{e}$, at a distance of 2 from $w$, which leaves all of the flats containing $e$ along the same ray at a distance of 1 . The rays $s_{e, f}$ and $s_{f, i}$, for flats $f$ containing $e$ also map to $r_{e}$, starting distances of 2 and 1 from $w$, respectively. The map $\phi$ is depicted in Fig. 2.

To check that $\phi$ is harmonic, we need to verify that locally, around each vertex $v$ of $\widetilde{\Gamma}_{M}$, the same number of edges map to each of the edges incident to $\phi(v)$, and this number is the degree of $\phi$ at $v$ [8, Sec. 2]. First, suppose that the vertex $v$ corresponds to an element $e^{\prime}$ other than $e$ and we have defined $\phi\left(e^{\prime}\right)$ to be the central vertex $w$. In this case, for each ray $r_{f}$ of $T$, there is exactly one edge incident to $e^{\prime}$ mapping to $r_{f}$, either the edge between $e^{\prime}$ and $f$ if $f$ contains $e$, or the unbounded edge $s_{e^{\prime}, f}$ if not. There is also exactly one edge mapping to $r_{e}$, which is the edge between $e^{\prime}$ and the unique flat containing both $e$ and $e^{\prime}$. Therefore, $\phi$ is harmonic at the vertex $e^{\prime}$ with local degree equal to 1 . 


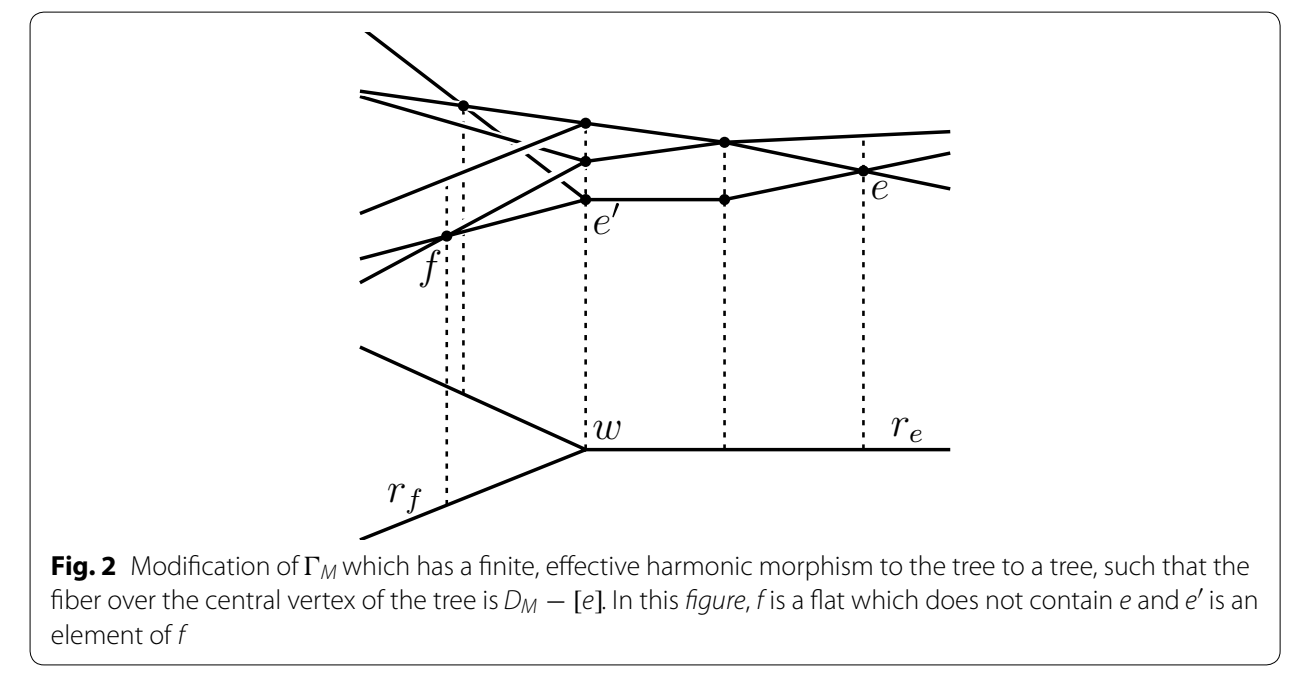

Second, at the vertex $e$, which maps along the edge $r_{e}$, there is one edge mapping to the bounded side of $r_{e}$ for each flat $f$ containing $e$ and also for each such flat, one infinite ray $s_{e, f}$ mapping to the unbounded side of $r_{e}$. Thus, $\phi$ is also harmonic at $e$, and has local degree equal to the number of flats containing $e$.

Finally, we check that $\phi$ is harmonic at a vertex corresponding to a flat $f$. If $f$ does not contain $e$, then there are $|f|$ rays mapping to the unbounded side of $r_{f}$ and the same number of edges mapping to the bounded side, connecting $f$ to the elements it contains. Thus, at such a vertex, $\phi$ is harmonic and its local degree is $|f|$. If $f$ does contain $e$, then there are $|f|-2$ rays mapping to the unbounded side of $r_{e}$ together with the edge connecting $f$ to $e$. On the bounded side of $r_{e}$, there are also $|f|-1$ edges, connecting $f$ to the elements $f \backslash\{e\}$, and so here $\phi$ is harmonic with local degree $|f|-1$.

To lift $\phi$ to a harmonic morphism of totally degenerate metrized complexes, we need to choose a map $\phi_{v}: \mathbb{P}^{1} \rightarrow \mathbb{P}^{1}$ for each vertex $v$ of $\widetilde{\Gamma}_{M}$ and an identification of the outgoing directions with points on $\mathbb{P}^{1}$. Having assumed characteristic not 2 , we can choose a tame homomorphism of degree equal to the degree of $\phi$ at $v$ as $\phi_{v}$. We identify the edges incident to $v$ with points of $\mathbb{P}^{1}$ at which $\phi_{\nu}$ is unramified, since these edges all have expansion factor equal to 1 . Then, the preimage of a $k$-point of the curve at $w$ consists of one point in each $\mathbb{P}^{1}$ corresponding to the elements $e^{\prime}$ not equal to $e$, because the local degrees at these vertices are equal to 1 . Thus, this preimage is a lift of $D_{M} \backslash[e]$ and we have our desired morphism of metrized complexes.

\section{Lifting matroid divisors}

In this section, we characterize the existence of lifts of matroid divisors in terms of realizability of the corresponding matroids. Recall from [6], that if $R$ is a discrete valuation ring with algebraically closed residue field, then a regular semistable family $\mathfrak{X}$ of curves over $R$ gives homomorphism $\rho$ from the group of divisors on the general fiber to the group of divisors on the dual graph $\Gamma$ of the special fiber. This dual graph is defined to have a vertex $v$ for each irreducible component of the special fiber and an edge for each point of intersection between two components. Then, for any divisor $\widetilde{D}$ on the general fiber of $\mathfrak{X}, \rho(\widetilde{D})$ is defined to be the formal sum of the vertices of $\Gamma$ with the coefficient 
of a vertex $v$ equal to the degree of the intersection of $\bar{D}$ with $C_{v}$, where $\bar{D}$ is the closure of $\widetilde{D}$ in $\mathfrak{X}$ and $C_{v}$ is the irreducible component corresponding to $v$ [6, Sec. 2A]. With this definition, we have an inequality $r(\widetilde{D}) \leq r(\rho(\widetilde{D}))$ between the algebraic and graph-theoretic ranks [6, Lem. 2.8].

We now consider the semistable family $\mathfrak{X}$ over a discrete valuation ring $R$, where we drop the assumption that the residue field of $R$ is algebraically closed. In this case, we apply the definitions from the previous paragraph by first base changing to a discretely valued extension $R^{\prime} \supset R$, such that the residue field of $R^{\prime}$ is algebraically closed and such that a uniformizer of $R$ is also a uniformizer of $R^{\prime}$. In particular, the dual graph has one vertex for each geometric irreducible component of the special fiber and it is independent of the choice of $R^{\prime}$. Also, the definition of $\rho(\widetilde{D})$ is independent of $R^{\prime}$ because it can be computed by taking the closure of $\widetilde{D}$ in $\mathfrak{X}$ and recording the degree of the pullback of this Cartier divisor to each of the geometric irreducible components of the special fiber. Moreover, for any family $\mathfrak{X}$, there is a finite étale extension $R^{\prime}$ of $R$ such that, after base changing to $R^{\prime}$, the irreducible components of the special fiber are geometrically irreducible. Therefore, after this base change, the computation of the dual graph and the specialization map can be carried out directly on the resulting family over $R^{\prime}$. Since the dimension of a linear system is invariant under base change, we also have a specialization inequality $r(\widetilde{D}) \leq r(\rho(\widetilde{D}))$ on $\mathfrak{X}$.

We will say that a lifting over $R$ of an effective divisor $D$ of rank $r$ on a graph $\Gamma$ is a regular semistable family $\mathfrak{X}$ over $R$ with dual graph $\Gamma$, together with an effective divisor $\widetilde{D}$ on the general fiber of $\mathfrak{X}$ such that $\rho(\widetilde{D})=D$ and $\widetilde{D}$ has rank $r$. Here, and throughout this section, a regular semistable family $\mathfrak{X}$ includes the hypothesis that $\mathfrak{X}$ is semistable after passing to an extension with algebraically closed residue field. The relationship between liftings of a matroid divisor $D_{M}$ and its matroid depends on the following, slightly weaker variant of realizability for $M$ :

Definition 3.1 Let $k$ be a field. We say that a matroid $M$ has a Galois-invariant realization over an extension of $k$ if there exists a finite scheme in $\mathbb{P}_{k}^{2}$ which becomes a union of distinct points over $\bar{k}$, and these points realize $M$.

Equivalently, a Galois-invariant realization is a realization over a finite Galois extension $k^{\prime}$ of $k$ such that the Galois group $\operatorname{Gal}\left(k^{\prime} / k\right)$ permutes the points of the realization. Thus, the distinction between a realization and a Galois-invariant realization is only relevant for matroids which have non-trivial symmetries. Moreover, Lemma 3.10 will show that any matroid can be extended to one where these symmetries can be broken, without affecting realizability over infinite fields.

Example 3.2 Let $M$ be the matroid determined by all 21 points of $\mathbb{P}_{\mathbb{F}_{4}}^{2}$. Then $M$ is not realizable over $\mathbb{P}_{\mathbb{F}_{2}}^{2}$ because it contains more than 7 elements, and there are only 7 points in $\mathbb{P}_{\mathbb{F}_{2}}^{2}$. However, $M$ is clearly realizable over $\mathbb{F}_{4}$ and the Galois group $\operatorname{Gal}\left(\mathbb{F}_{4} / \mathbb{F}_{2}\right) \cong \mathbb{Z} / 2$ acts on these points by swapping pairs. Thus, $M$ has a Galois-invariant realization over an extension of $\mathbb{F}_{2}$. 
Example 3.3 Let $M$ be the Hesse matroid of 9 elements and 12 flats. Then $M$ is not realizable over $\mathbb{R}$ by the Sylvester-Gallai theorem. However, the flex points of any elliptic curve are a realization of $M$ over $\mathbb{C}$. If the elliptic curve is defined over $\mathbb{R}$, then the set of all flexes points is also defined over $\mathbb{R}$, so $M$ has a Galois-invariant realization over an extension of $\mathbb{R}$.

Theorem 3.4 Let $\Gamma_{M}$ and $D_{M}$ be the graph and divisor obtained from a rank 3 simple matroid $M$ as in Sect. 2. Also, let $R$ be any discrete valuation ring with residue field $k$. If $D_{M}$ lifts over $R$, then the matroid $M$ has a Galois-invariant realization over an extension of $k$.

By projective duality, a point in $\mathbb{P}^{2}$ is equivalent to a line in the dual projective space $\mathbb{P}^{2}$. Thus, the collection of points realizing a matroid is equivalent to a collection of lines, in which the flats correspond to the points of common intersection. It is this dual representation that we will construct from the lifting.

Proof of Theorem 3.4 Let $\mathfrak{X}$ be the semistable family over $R$ and $\widetilde{D}$ a rank 2 divisor on the general fiber of $\mathfrak{X}$ with $\rho(\widetilde{D})=D_{M}$. First, we make the simplifying assumption that the components of the special fiber are geometrically irreducible, so that we can compute specializations in $\mathfrak{X}$, without needing to take further field extensions. Let $\bar{D}$ denote the closure in $\mathfrak{X}$ of $\widetilde{D}$. By assumption, $H^{0}(\mathfrak{X}, \mathcal{O}(\bar{D}))$ is isomorphic to the free $R$-module $R^{3}$. By restricting a basis of these sections to the special fiber $\mathfrak{X}_{0}$, we have a rank 2 linear series on the reducible curve $\mathfrak{X}_{0}$.

If $\bar{D}$ intersected a node of $\mathfrak{X}_{0}$, then it would intersect both components of $\mathfrak{X}_{0}$ containing that node, so $\rho(D)$ would have positive multiplicity on two adjacent vertices. However, $\Gamma_{M}$ is bipartite and the divisor $D_{M}$ is supported on one of these parts, corresponding to the elements of the matroid, so $\bar{D}$ cannot intersect any of the nodes of $\mathfrak{X}_{0}$. Thus, the base locus of our linear series consists of a finite number of smooth points of $\mathfrak{X}_{0}$. Since the base locus consists of smooth points, we can subtract the base points to get a regular, non-degenerate morphism $\phi: \mathfrak{X}_{0} \rightarrow \mathbb{P}_{k}^{2}$.

By the assumption that $\widetilde{D}$ specializes to $D_{M}$, we have an upper bound on the degree of $\phi$ restricted to each component of $\mathfrak{X}_{0}$. For a flat $f$ of $M$, the corresponding component $C_{f}$ has degree 0 under $\phi$, so $\phi\left(C_{f}\right)$ consists of a single point. For an element $e$, the corresponding component $C_{e}$ has either degree 1 or 0 depending on whether the intersection of $\bar{D}$ with $C_{e}$ is contained in the base locus. If the intersection is in the base locus, then $C_{e}$ again maps to a point, and if not, $C_{e}$ maps isomorphically to a line in $\mathbb{P}_{k}^{2}$. Thus, the image $\phi\left(\mathfrak{X}_{0}\right)$ is a union of lines in $\mathbb{P}_{k}^{2}$, which we will show to be a dual realization of the matroid $M$. Let $f$ be a flat of $M$. Since the component of $\mathfrak{X}_{0}$ corresponding to $f$ maps to a point, the images of the components corresponding to the elements in $f$ all have a common point of intersection.

Now let $e_{1}$ be an element of $M$ and suppose that the component $C_{e_{1}}$ maps to a point $\phi\left(C_{e_{1}}\right)$. Since every other element $e^{\prime}$ is in a flat with $e_{1}$, that means that $\phi\left(C_{e^{\prime}}\right)$, the image of the corresponding component must contain the point $\phi\left(C_{e_{1}}\right)$. Since $\phi$ is non-degenerate, there must be at least one component $C_{e_{2}}$ which maps to a line. Let $e_{3}$ be an element of $M$ which completes $\left\{e_{1}, e_{2}\right\}$ to a basis. Thus, the flat containing $e_{2}$ and $e_{1}$ is distinct 
from the flat containing $e_{2}$ and $e_{3}$. Since $\phi$ maps $C_{e_{2}}$ isomorphically onto its image, this means that $\phi\left(C_{e_{3}}\right)$ must meet $\phi\left(C_{e_{2}}\right)$ at a point distinct from the point $\phi\left(C_{e_{1}}\right)$. Thus, $\phi\left(C_{e_{3}}\right)$ must be equal to $\phi\left(C_{e_{2}}\right)$. Any other element $e^{\prime \prime}$ in $M$ forms a basis with $e_{1}$ and either $e_{2}$ or $e_{3}$ (or both). In either case, the same argument again shows that $C_{e^{\prime \prime}}$ must map to the same line as $C_{e_{2}}$ and $C_{e_{3}}$. Thus, this line would be the entire image of $\phi$, which again contradicts the assume non-degeneracy. Therefore, we conclude that $\phi$ maps each component $C_{e}$ corresponding to an element $e$ isomorphically onto a line in $\mathbb{P}_{k}^{2}$. We have already shown that for any set of elements in a flat, the corresponding lines intersect at the same point. Moreover, because each component $C_{e}$ maps isomorphically onto its image, distinct flats must correspond to distinct points in $\mathbb{P}_{k}^{2}$. Thus, $\phi\left(\mathfrak{X}_{0}\right)$ is a dual realization of the matroid $M$.

If the components of the special fiber are not geometrically irreducible, then we can find a finite étale extension $R^{\prime}$ of $R$ over which they are. In our construction of a realization of $M$ over the residue field of $R^{\prime}$, we can assume that we have chosen a basis of $H^{0}\left(\mathfrak{X} \times R_{R}, \mathcal{O}\right)$ that is defined over $R$. Then, the matroid realization will be the base extension of a map of $k$-schemes $\mathfrak{X}_{0} \rightarrow \mathbb{P}_{k}^{2}$. We let $k^{\prime}$ be the Galois closure of the residue field of $R^{\prime}$. Then $\operatorname{Gal}\left(k^{\prime} / k\right)$ acts on the realization of $M$ over $k^{\prime}$, but the total collection of lines is defined over $k$, and thus invariant. Thus, $M$ has a Galois-invariant realization over an extension of $k$ as desired.

For the converse of Theorem 3.4, we need to consider realizations of matroids over discrete valuations ring $R$, by which we mean $R$-points in $\mathbb{P}^{2}$ whose images in both the residue field and the fraction field realize $M$. For example, if $R$ contains a field over which $M$ is realizable, then $M$ is realizable over $R$. We say that $M$ has a Galois-invariant realization over an extension of $R$ if there exists a finite, flat scheme in $\mathbb{P}_{R}^{2}$ whose special and general fibers are Galois-invariant realizations of $M$ over extensions of the residue field and fraction field of $R$, respectively.

In the following theorem, a complete flag refers to the pair of an element $e$ and a flat $f$ such that $e$ is contained in $f$.

Theorem 3.5 Let $R$ be a discrete valuation ring with residue field $k$. Let $M$ be a simple rank 3 matroid with a Galois-invariant realization over an extension of $R$. Assume that $|k|>m-2 n+1$, where $n$ is the number of elements of $M$ and $m$ is the number of complete flags. Then $\Gamma_{M}$ and $D_{M}$ lift over $R$.

Note that Theorem 3.5 does not make any completeness or other assumptions on the DVR beyond the cardinality of the residue field. In contrast, ignoring $D_{M}$ and its rank, a semistable model $\mathcal{X}$ is only known to exist for an arbitrary graph when the valuation ring is complete [6, Thm. B.2].

We construct the semistable family in Theorem 3.5 using a blow-up of projective space. We begin with a computation of the Euler characteristic for this blow-up.

Lemma 3.6 Let $S$ be the blow-up of $\mathbb{P}_{K}^{2}$ at the points of intersection of an arrangement of $n$ lines. If $A$ is the union of the strict transforms of the lines and the exceptional divisors, then the dimension of $H^{0}(S, \mathcal{O}(A))$ is at least $2 n+1$. 
Proof We first use Riemann-Roch to compute that $\chi(\mathcal{O}(A))$ is $2 n+1$. Let $m$ be the number of complete flags of $M$, the matroid of the line arrangement $A$. We let $H$ denote the pullback of the class of a line on $\mathbb{P}^{2}$ and $C_{f}$ to denote the exceptional lines. Then, we have the following linear equivalences

$$
\begin{aligned}
& A \sim n H-\sum_{f}(|f|-1) C_{f} \\
& K_{S} \sim-3 H+\sum_{f} C_{f}
\end{aligned}
$$

Now, Riemann-Roch for surfaces tells us that

$$
\begin{aligned}
\chi(\mathcal{O}(A))=\frac{A^{2}-A \cdot K_{S}}{2}+1 & =\frac{n^{2}-\sum_{f}(|f|-1)^{2}+3 n-\sum_{f}(|f|-1)}{2}+1 \\
& =\frac{n^{2}+3 n-\sum_{f}|f|(|f|-1)}{2}+1 .
\end{aligned}
$$

We can think of the summation $\sum_{f}|f|(|f|-1)$ as an enumeration of all triples of a flat and two distinct elements of the flat. Since two distinct elements uniquely determine a flat, we have the identity that $\sum_{f}|f|^{2}=n(n-1)$, so (2) simplifies to $\chi(\mathcal{O}(A))=2 n+1$.

It now suffices to prove that $H^{2}(S, \mathcal{O}(A))$ is zero, which is equivalent, by Serre duality, to showing that $K_{S}-A$ is not linearly equivalent to an effective divisor. The pushforward of $K_{S}-A$ to $\mathbb{P}^{2}$ is $-(n+3) H$, which is not linearly equivalent to an effective divisor, and thus $H^{2}(S, \mathcal{O}(A))$ must be zero. Therefore,

$$
\chi(\mathcal{O}(A))=H^{0}(S, \mathcal{O}(A))-H^{1}(S, \mathcal{O}(A)) \leq H^{0}(S, \mathcal{O}(A))
$$

which together with the computation above yields the desired inequality.

Proof of Theorem 3.5 We first assume that $M$ is realizable over $R$, and then at the end, we will handle Galois-invariant realizations over extensions. Thus, we can fix a dual realization of $M$ as a set of lines in $\mathbb{P}_{R}^{2}$, and let $S$ be the blow-up of $\mathbb{P}_{R}^{2}$ at all the points of intersections of the lines. We let the divisor $A \subset S$ be the sum of the strict transforms of the lines and the exceptional divisors. Note that $A$ is a simple normal crossing divisor whose dual complex is $\Gamma_{M}$. As in the proof of Theorem 3.4, we denote the components of $A$ as $C_{f}$ and $C_{e}$ corresponding to a flat $f$ and an element $e$ of $M$, respectively.

We claim that $A$ is a base-point-free divisor on $S$. Any two lines of the matroid configuration are linearly equivalent in $\mathbb{P}_{R}^{2}$. The preimage of a linear equivalence between lines corresponding to elements $e$ and $e^{\prime}$ is the divisor:

$$
\left[C_{e^{\prime}}\right]-\left[C_{e}\right]+\sum_{f: e^{\prime} \in f, e \notin f}\left[C_{f}\right]-\sum_{f: e \in f, e^{\prime} \notin f}\left[C_{f}\right] .
$$

Thus, we have a linear equivalence between $A$ and a divisor which does not contain $C_{e}$, nor $C_{f}$ for any of the flats containing $e$ but not $e^{\prime}$. By varying $e$ and $e^{\prime}$, we get linearly equivalent divisors whose common intersection is empty.

We now look for a function $g \in H^{0}(S, \mathcal{O}(A)) \otimes_{R} k$ which does not vanish at the nodes of $A$. For each of the $m$ nodes, the condition of vanishing at that node amounts 
to one linear condition on $H^{0}(S, \mathcal{O}(A)) \otimes_{R} k$. Since $A$ is base-point free, this is a nontrivial linear condition, defining a hyperplane. Moreover, because of the degrees of the intersection of $A$ with its components, the only functions vanishing on all of the nodes are multiples of the defining equation of $A$. If the residue field is sufficiently large, then we can find an element $g \in H^{0}(S, \mathcal{O}(A)) \otimes_{R} k$ avoiding these hyperplanes, and $|k|>m-2 n+1$ is sufficient by Lemmas 3.6 and 3.7. Now we lift $g$ to $\widetilde{g} \in H^{0}(S, \mathcal{O}(A))$, and set $\mathfrak{X}$ to be the scheme defined by $h+\pi \widetilde{g}$, where $h$ is the defining equation of $A$ and $\pi$ is a uniformizer of $R$. It is clear that $\mathfrak{X}$ is a flat family of curves over $R$ whose special fiber is $A$ and thus has dual graph $\Gamma_{M}$. It remains to check that $\mathfrak{X}$ is regular and for this it is sufficient to check the nodes of $A_{k}$. In the local ring of a node, $h$ is in the square of the maximal ideal, but by construction $\pi \widetilde{g}$ is not, and thus, at this point $\mathfrak{X}$ is regular.

Finally, we can take $D$ to be the preimage of any line in $\mathbb{P}_{R}^{2}$ which misses the points of intersection. Again, by Lemma 3.7 below, it is sufficient that $|k|>\ell-2$, where $\ell$ is the number of flats. We claim that $m-2 n+1 \geq \ell-2$, and we have assumed that $|k|>m-2 n+1$. This claimed inequality can be proved using induction similar to the proof of Theorem 4.1, but it also follows from Riemann-Roch for graphs [7, Thm. 1.12]. Since $\Gamma_{M}$ has genus $m-\ell-n+1$, then the Riemann-Roch inequality tells us that

$$
2=r\left(D_{M}\right) \geq n-(m-\ell-n+1)=\ell-m+2 n-1,
$$

which is equivalent to the claimed inequality.

Now, we assume that $M$ may only have a Galois-invariant realization over an extension of $R$. We can construct the blow-up $S$ in the same way, since the singular locus of the line configuration is defined over $R$. Again, the divisor $A$ is base-point free, because we have already checked that it is base point free after passing to an extension where the lines are defined. Finally, we need to choose the function $g$ and the line which pulls back to $D$ by avoiding certain linear conditions defined over an extension of $k$. However, when restricted to $k$, these remain linear conditions, possibly of higher codimension, so we can again avoid them under our hypothesis on $|k|$.

Lemma 3.7 Let $H_{1}, \ldots, H_{m}$ be hyperplanes in the vector space $k^{N}$, where $k$ is a field. Let $c$ denote the codimension of the intersection $H_{1} \cap \cdots \cap H_{m}$. If $k$ is infinite or if $k$ is the finite field with $q$ elements and $q>m-c+1$, then there exists a point in $k^{N}$ not contained in any hyperplane.

Proof If $k$ is infinite, the statement is clear, so we assume that $k$ is finite with $q$ elements. We first quotient out by the intersection $H_{1} \cap \cdots \cap H_{m}$, so we are working in a vector space of dimension $c$ and we know that no non-zero vector is contained in all hyperplanes. This means that the vectors defining the hyperplanes span the dual vector space, so we can choose a subset as a basis. Thus, we assume that the first $c$ hyperplanes are the coordinate hyperplanes. The complement of these consists of all vectors with non-zero coordinates, of which there are $(q-1)^{c}$. Each of the remaining $m-c$ hyperplanes contains at most $(q-1)^{c-1}$ of these. Our assumption is that $q-1>m-c$, so there must be at least one point not contained in any of the hyperplanes.

We illustrate Theorems 3.4 and 3.5 and highlight the difference between their conditions with the following two examples. 
Example 3.8 Let $M$ be the Fano matroid, which whose realization in $\mathbb{P}_{\mathbb{F}_{2}}^{2}$ consists of all $7 \mathbb{F}_{2}$-points. Then $M$ is realizable over a field if and only if the field has equicharacteristic 2. Thus, by Theorem 3.4, a necessary condition for $\Gamma_{M}$ and $D_{M}$ to lift over a valuation ring $R$ is that the residue field of $R$ has characteristic 2 . On the other hand, $M$ has 7 elements and 21 complete flags, so Theorem 3.5 says that if $R$ has equicharacteristic 2 and the residue field of $R$ has more than 8 elements, then $\Gamma_{M}$ and $D_{M}$ lift over $R$. We do not know if there exists a lift of $\Gamma_{M}$ and $D_{M}$ over any valuation ring of mixed characteristic 2 .

Example 3.9 One the other hand, let $M$ be the non-Fano matroid, which is realizable over $k$ if and only $k$ has characteristic not equal to 2 . Moreover, $M$ is realizable over any valuation ring $R$ in which 2 is invertible. Thus, $\Gamma_{M}$ and $D_{M}$ lift over a valuation ring $R$, only if the residue field of $R$ has characteristic different than 2 by Theorem 3.4. The converse is true, so long as the residue field has more than 11 elements by Theorem 3.5.

Since Theorems 3.4 and 3.5 refer to Galois-invariant realizations, we will need the following lemma to relate such realizations with ordinary matroid realizations.

Lemma 3.10 Let $M$ be a matroid of rank 3. Then there exists a matroid $M^{\prime}$ such that for any infinite field $k$, the following are equivalent:

1. $M$ has a realization over $k$.

2. $M^{\prime}$ has a realization over $k$.

3. $M^{\prime}$ has a Galois-invariant realization over an extension of $k$.

Proof We use the following construction of an extension of a matroid. Suppose that $M$ is a rank 3 matroid and $f$ is a flat of $M$. We construct a matroid $M^{\prime \prime}$ which contains the elements of $M$, together with an additional element $x$. The flats of $M^{\prime \prime}$ are those of $M$, except that $f$ is replaced by $f \cup\{x\}$, and two-element flats for $x$ and every element not in $f$. By repeating this construction, we can construct a matroid $M^{\prime}$ such every flat which comes from one of the flats of $M$ has a different number of elements.

Now we prove that the conditions in the lemma statement are equivalent for this choice of $M^{\prime}$. First, assume that $M$ has a realization over an infinite field $k$. We can inductively extend this to a realization of $M^{\prime}$. At each step, when adding an element $x$ as above, it is sufficient to place $x$ at a point along the line corresponding to $f$ such that it does not coincide with any of the other points, and it is not contained in any of the lines spanned by two points not in $f$. We can choose such a point for $x$ since $k$ is infinite. Second, if $M^{\prime}$ has a realization over $k$, then by definition, it has a Galois-invariant realization over an extension of $k$.

Finally, we suppose that $M^{\prime}$ has a Galois-invariant realization over an extension of $k$ and we want to show that $M$ has a realization over $k$. Suppose we have a realization over a Galois extension $k^{\prime}$ of $k$. Since all the flats from the original matroid contain different numbers of points, the Galois group does not permute the corresponding lines in the realization. Therefore, the lines and thus also the points from the original matroid $M$ must be defined over $k$. Therefore, the restriction of this realization gives a realization of $M$ over $k$, which completes the proof of the lemma. 
Proof of Theorem 1.3 As in the statement of the theorem, let $X$ be a scheme of finite type over $\mathbb{Z}$. We choose an affine open cover of $X$ and let $\widetilde{X}$ be the disjoint union of these affine schemes. By the scheme-theoretic version of Mnëv's universality theorem, either Theorem 1.14 in [17] or our Theorem 5.3, there is a matroid $M$ of rank 3 whose realization space is isomorphic to an open subset $U$ of $\widetilde{X} \times \mathbb{A}^{N}$ and $U$ maps surjectively onto $X$. Now let $M^{\prime}$ be the matroid as in Lemma 3.10 and we claim that $\Gamma_{M^{\prime}}$ and $D_{M^{\prime}}$ have the desired properties for the theorem.

Let $k$ be any infinite field, and then $X$ clearly has a $k$-point if and only if $\widetilde{X}$ has a $k$-point. Likewise, since $k$ is infinite, any non-empty subset of $\mathbb{A}_{k}^{N}$ has a $k$-point, so $U$ also has a $k$-point if and only if $X$ has a $k$-point. By Lemma 3.10, these conditions are equivalent to $M^{\prime}$ having a Galois-invariant realization over an extension of $k$. Supposing that $X$ has a $k$-point and thus $M^{\prime}$ has a realization over $k$, then $\Gamma_{M^{\prime}}$ and $D_{M^{\prime}}$ have a lifting over $k[[t]]$ by Theorem 3.5. Conversely, if $D_{M^{\prime}}$ has a lifting over $k[[t]]$, then $M^{\prime}$ has a Galois-invariant realization over an extension of $k$ by Theorem 3.4, and thus $M$ has a realization over $k$ by Lemma 3.10, so $X$ has a $k$-point.

\section{Brill-Noether theory}

In this section, we take a detour and look at connections to Brill-Noether theory and the analogy between limit linear series and tropical divisors. In the theory of limit linear series, a key technique is the observation that if the moduli space of limit linear series on the degenerate curve has the expected dimension then it lifts to a linear series [13, Thm. 3.4]. Here, the expected dimension of limit linear series of degree $d$ and rank $r$ on a curve of genus $g$ is $\rho(g, r, d)=g-(r+1)(g+r-d)$. It is natural to ask if a tropical analog of this result is true: if the dimension of the moduli space of divisor classes on a tropical curve of degree $d$ and rank at least $r$ has (local) dimension $\rho(g, r, d)$, then does every such divisor lift? See [10] for further discussion and one case with an affirmative answer. The main result of this section is that the matroid divisors and graphs constructed in this paper do not provide a negative answer to the above question.

We begin with the following classification:

Theorem 4.1 Let $M$ be a rank 3 simple matroid, with $g$ and $d$ equal to the genus of $\Gamma_{M}$ and degree of $D_{M}$, respectively. If $\rho=\rho(g, 2, d) \geq 0$, then $M$ is one of the following matroids:

1. The one-element extension of the uniform matroid $U_{2, n-1}$, with $\rho=n-2$.

2. The uniform matroid $U_{3,4}$ with $\rho=0$.

3. The matroid defined by the vectors: $(1,0,0),(1,0,1),(0,0,1),(0,1,1),(0,1,0)$, with $\rho=1$.

4. The matroid in the previous example together with $(1,0, \lambda)$ for any element $\lambda$ of the field other than 1 and 0 , with $\rho=0$.

5. The matroid consisting of the point of intersection between any pair in a collection of 4 generic lines, for which we can take the coordinates to be the vectors from (3) together with $(1,1,1)$, with $\rho=0$.

The last three cases of Theorem 4.1 are illustrated in Fig. 3. 


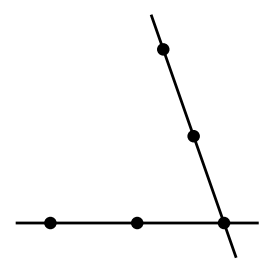

(3)

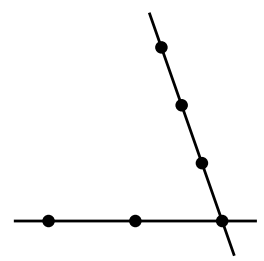

(4)

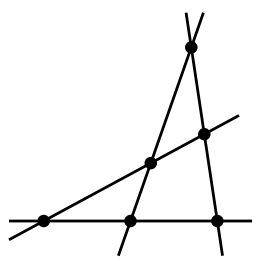

(5)

Fig. 3 Matroids from Theorem 4.1

Proof We first compute the invariants for the graph $\Gamma_{D}$ and divisor $D_{M}$ constructed in Sect. 2. As before, we let $n$ be the number of elements of $M, \ell$ the number of flats, and $m$ the number of complete flags. Since $\Gamma_{D}$ consists of $m$ edges and $n+\ell$ vertices, it has genus $m-n-\ell+1$. It is also immediate from its definition that $D_{M}$ has degree $n$. Thus, the expected dimension of rank 2 divisors is

$$
\rho=m-n-\ell+1-3((m-n-\ell+1)+2-n)=5 n+2 \ell-2 m-8
$$

Now, assume that $\rho$ is non-negative for $M$ and we consider what happens to $\rho$ when we remove a single element $e$ from a matroid, where $e$ is not contained in all bases. For every flat containing $e$, we decrease the number of complete flags by 1 if that flat contains at least 3 elements, and if it contains 2 elements, then we decrease the number of flags by 2 and the number of flats by 1 . Thus, by (3), $\rho$ drops by $5-2 s$, where $s$ is the number of flats in $M$ which contain $e$. Since $e$ must be contained in at least 2 flats, either $M \backslash e$, the matroid formed by removing $e$ has positive $\rho$ or $e$ is contained in exactly 2 flats.

We first consider the latter case, in which $e$ is contained in exactly two flats, which we assume to have cardinality $a+1$ and $b+1$, respectively. The integers $a$ and $b$ completely determine the matroid because all the other flats consist of a pair of elements, one from each of these sets. Thus, there are $a b+2$ flats and $2 a b+a+b+2$ complete flags. Using (3), we get

$$
\rho=5(a+b+1)+2(a b+2)-2(2 a b+a+b+2)-8=-2 a b+3 a+3 b-3 .
$$

One can check that, up to swapping $a$ and $b$, the only non-negative values of this expression are when $a=1$ and $b$ is arbitrary or $a=2$ and $b$ is 2 or 3 . These correspond to cases (1), (3), and (4), respectively, from the theorem statement.

Now we consider the case that $e$ contained in more than two flats, in which case $M \backslash e$ satisfies $\rho>0$. By induction on the number of elements, we can assume that $M \backslash e$ is on our list, in which case the possibilities with $\rho>0$ are (1) and case (3). For the former matroid, if $e$ is contained in a flat of $M \backslash e$, then $M$ is a matroid of the type from the previous paragraph, with $a$ equal to 1 or 2 . On the other hand, if $e$ contained only in 2-element flats, then $e$ is contained in $n-1$ flats, so

$$
\rho(M)=\rho(M \backslash e)+5-2(n-1)=(n-3)+7-2 n=4-n .
$$

The only possibility is $n=4$, for which we get (2), the uniform matroid. Finally, if $M \backslash e$ is the matroid in case (3), then the only relevant possibilities are those for which $e$ is contained in at most 3 flats, for which the possible matroids are (4) or (5). 
Proposition 4.2 If $R$ is a DVR and $M$ is one of the matroids in Theorem 4.1, then $M$ has a Galois-invariant realization over an extension of $R$.

Proof The matroids (2), (3) and (5) are regular matroids, i.e., realizable over $\mathbb{Z}$, so they are a fortiori realizable over any DVR. Moreover, the other matroids in case (1) and (4) are realizable over $R$ so long as the residue field has at least $n-2$ and 3 elements, respectively. We will show that if the residue field is finite, then the one-element extension of $U_{2, n-1}$ has a Galois-invariant realization over $R$. The other case is similar.

Let $M$ be the one-element extension of $U_{2, n-1}$ and suppose the residue field $k$ is finite. We choose a polynomial with coefficients of degree $n-1$ in $R$ whose reduction to $k$ is square free. Adjoining the roots of this polynomial defines an unramified extension $R^{\prime}$ of $R$, and we write $a_{1}, \ldots, a_{n-1}$ for its roots in $R$. Then, the vectors $\left(1, a_{1}, 0\right), \ldots,\left(1, a_{n-1}, 0\right),(0,0,1)$ give a Galois-invariant realization of $M$ over $R^{\prime}$, which is what we wanted to show.

\section{Quantitative Mnëv universality}

In this section, we prove a quantitative version of Mnëv universality over Spec $\mathbb{Z}$ with Theorem 1.5 as our desired application. We follow the strategy of [17, Thm. 1.14], but use the more efficient building blocks used in, for example, [18]. We pay close attention to the number of points used in our construction to get effective bounds on the degree of the corresponding matroid divisor. These bounds are expressed in terms of the following representation.

Definition 5.1 Let $S_{n}$ denote the polynomial ring $\mathbb{Z}\left[y_{1}, \ldots, y_{n}\right]$. In the extension $S_{n}[t]$, we also introduce the coordinates $x_{i}$ defined by $x_{0}=t$ and $x_{i}=y_{i}+t$ for $1 \leq i \leq n$. In addition, for $n<i \leq m$, suppose we have elements $x_{i} \in S_{n}[t]$ such that:

1. Each $x_{i}$ is defined as one of $x_{i}=x_{j}+x_{k}, x_{i}=x_{j} x_{k}$, or $x_{i}=x_{j}+1$, where $j, k<i$.

2. Each $x_{i}$ is monic as a polynomial in $t$ with coefficients in $S_{n}$.

The coordinates $x_{i}$ for $1 \leq i \leq n$ will be called free variables and the three operations for defining new variables in (1) will be called addition, multiplication, and incrementing, respectively.

Moreover, we suppose we have finite sets of equalities $E$ and inequalities $I$ consisting of pairs $(i, j)$ such that $x_{i}-x_{j}$ is in $S_{n} \subset S_{n}[t]$. We then say that the algebra:

$$
S_{n}\left[\left(x_{i^{\prime}}-x_{j^{\prime}}\right)^{-1}\right]_{\left(i^{\prime} j^{\prime}\right) \in I} /\left\langle x_{i}-x_{j} \mid(i, j) \in E\right\rangle
$$

has an elementary monic representation consisting of the above data, namely, the integers $n$ and $m$, the expression of each $x_{i}$ as an addition, multiplication, or increment for $n<i \leq m$, and the sets of equalities and inequalities.

The inequalities $I$ in Definition 5.1 are not strictly necessary because an inverse to $x_{i}-x_{j}$ can always be introduced as a new variable, but the direct use of inequalities may be more efficient, such as in the proof of Theorem 1.5. 
Proposition 5.2 There exists an elementary monic representation of any finitely generated $\mathbb{Z}$-algebra.

Proof We begin by presenting the $\mathbb{Z}$-algebra $R$ as

$$
R=\mathbb{Z}\left[y_{1}, \ldots, y_{n}\right] /\left\langle f_{1}-g_{1}, \ldots, f_{m}-g_{m}\right\rangle,
$$

where each polynomial $f_{k}$ and $g_{k}$ has positive integral coefficients. Then $f_{k}$ and $g_{k}$ can be constructed by a sequence of multiplication and addition operations applied to the variables $y_{i}$ and the constant 1 . Obviously, we can assume that our multiplication never involves the constant 1 . To get an elementary monic representation, we first replace the variables $y_{i}$ with $x_{i}=y_{i}+t$ in the constructions of $f_{k}$ and $g_{k}$, with some adjustments, as follows. Since Definition 5.1 does not allow addition of 1 with itself, we replace such operations by first introducing a new variable $x_{i}=x_{0}+1=t+1$ and then adding 1 to $x_{i}$. Similarly, to satisfy the second condition of Definition 5.1, when adding two variables $x_{i}$ and $x_{j}$ which are both monic of the same degree $d$ in $t$, we first compute an intermediate $x_{i^{\prime}}=t^{d+1}+x_{i}$ and then the sum $x_{i^{\prime}}+x_{j}=t^{d+1}+x_{i}+x_{j}$. In this way, we ensure that all of the $x_{i}$ variables are monic in $t$. Moreover, $x_{i}$ and $x_{j}$ agree with $f_{k}$ and $g_{k}$, respectively, modulo $t$, but to be able to have an equality $x_{i}=x_{j}$ in an elementary monic representation, the difference $x_{i}-x_{j}$ have to not involve $t$.

Therefore, we want to replace $x_{i}$ and $x_{j}$ by polynomials $x_{i}^{\prime}$ and $x_{j}^{\prime}$ which agree with $x_{i}$ and $x_{j}$ modulo $t$, but such that the difference $x_{i}^{\prime}-x_{j}^{\prime}$ does not involve $t$. We do this by double induction, first, on the maximum total degree in the $y$ variables of the terms of $x_{i}-x_{j}$ that involve $t$, and second, on the number of terms of that degree.

Thus, we suppose that $c t^{s} y_{1}^{a_{1}} \cdots y_{n}^{a_{n}}$ is a term of $x_{i}-x_{j}$ whose total degree in the $y$ variables is maximal among terms with $s>0$. By swapping $i$ and $j$ if necessary, we can assume that $c$ is positive. We then use multiplication operations to construct:

$$
x_{\ell}=t^{s} x_{1}^{a_{1}} \cdots x_{n}^{a_{n}}=t^{s}\left(t+y_{1}\right)^{a_{1}} \cdots\left(t+y_{n}\right)^{a_{n}}=t^{s} y_{1}^{a_{1}} \cdots y_{m}^{a_{m}}+\cdots,
$$

where the final ellipsis denotes omitted terms with lower degree in the $y$ variables. First suppose that $x_{j}$ and $x_{\ell}$ have different degrees in the $t$ variable. Then, we can use $c$ addition operations to construct $x_{j^{\prime}}=x_{j}+c x_{\ell}$, and we set $i^{\prime}=i$. On the other hand, if $x_{j}$ and $x_{\ell}$ have the same degree in $t$, then let $d$ be an integer larger than the $t$-degree of $x_{i}, x_{j}$, and $x_{\ell}$, and we use additions to construct $x_{j^{\prime}}=x_{j}+c x_{\ell}+t^{d}$ and $x_{i^{\prime}}=x_{i}+t^{d}$. In either case, $x_{i^{\prime}}$ and $x_{j^{\prime}}$ equal $x_{i}$ and $x_{j}$, respectively, modulo $t$. Moreover, the term $c t^{s} y_{1}^{a_{1}} \cdots y_{m}^{a_{m}}$ has been eliminated from $x_{i^{\prime}}-x_{j^{\prime}}$, while only introducing new terms which have lower degree in the $y$ variables. Thus, by induction, we can eliminate all terms of $x_{i}-x_{j}$ which involve $t$ and have maximal total degree in the $y$ variables among such terms, and by the second level of induction, we can eliminate such terms in all degrees. We, therefore, have constructions of variables $x_{i^{\prime \prime}}$ and $y_{j^{\prime \prime}}$ such that $x_{i^{\prime \prime}}-x_{j^{\prime \prime}}=f_{k}-g_{k}$. Setting these equal for $k=1, \ldots, m$ gives us the elementary monic representation of $R$.

Given a matroid $M$, its possible realizations form a scheme, called the realization space of the matroid [15, Sec. 9.5]. Explicitly, given a rank 3 matroid with $n$ elements, each flat of the matroid defines a closed, determinantal condition in $\left(\mathbb{P}_{\mathbb{Z}}^{2}\right)^{n}$ and each triple of elements which is not in any flat defines an open condition by not being collinear. The realization space is the quotient by $\mathrm{PGL}_{3}(\mathbb{Z})$ of the scheme-theoretic intersection of these 
conditions. We will only consider the case when this action is free, in which case the quotient will be an affine scheme over Spec $\mathbb{Z}$.

Theorem 5.3 (Mnëv universality) For any finite-type $\mathbb{Z}$-algebra $R$, there exists a rank 3 matroid $M$ whose realization space is an open subset $U \subset \mathbb{A}^{N} \times$ Spec $R$ such that $U$ projects surjectively onto $\operatorname{Spec} R$.

Moreover, if $R$ has an elementary monic representation with $n$ free variables, a additions, $m$ multiplications, o increments, e equalities, and $i$ inequalities, then $M$ has

$$
3 n+7 a+7 o+6 m+5 e+6 i+6
$$

elements, and

$$
N=3(n+a+o+m+e+i)+1
$$

Proof By Proposition 5.2, we can assume that $R$ has an elementary monic representation. Both the matroid and its potential realization will be built up from the elementary monic representation, beginning with the free variables and then applying the addition, multiplication, and increment operations. We describe the constructions of both the matroid and the realization in parallel for ease of explaining their relationship.

We begin with the free variables. For $x_{0}=t$ and for each free variable $x_{i}$ of the representation, we have a line, realized generically, passing through a common fixed point. In the figures below, we will draw these horizontally so that the common point is at infinity. On each of these lines we have 3 additional points, whose positions along the line are generic. Our convention will always be that points whose relative position is not specified are generic. In other words, unless otherwise specified to lie on a line, each pair of points correspond to a 2-element flat.

From each set of 4 points on one of these free variable lines, we can take the crossratio, which is invariant under the action of $\mathrm{PGL}_{3}(\mathbb{Z})$. Therefore, by taking the crossratio on each line as the value for the corresponding coordinate $x_{i}=t+y_{i}$ or $x_{0}=t$, we define a morphism from the realization space of the matroid defined thus far to $\mathbb{A}^{n+1}=\operatorname{Spec}_{n}[t]$, where $S_{n}=\mathbb{Z}\left[y_{1}, \ldots, y_{n}\right]$ as in Definition 5.1. Our goal with the remainder of the construction is to constrain the realization such that the projection to $\operatorname{Spec} S_{n}$ is surjective onto $\operatorname{Spec} R \subset \operatorname{Spec} S_{n}$.

Concretely, the cross-ratio is the position of one point on the line in coordinates where the other points are at 0,1 , and $\infty$. For us, the point common to all variable lines will be at $\infty$, so we will refer to the other points along the line as the " 0 " point, the " 1 " point, and the variable point. We will next embed the operations of addition, multiplication, and incrementing from the elementary monic representation. The result of each of these operations will be encoded as the cross-ratio of 4 points on a generic horizontal line, in the same way as with the free variables.

First, multiplication of distinct variables $x_{i}=x_{j} x_{k}$ is constructed as in Fig. 4, where the $x_{j}$ and $x_{k}$ lines refer to the lines previously constructed for those variables and the other points are new. We can choose the horizontal line for $x_{i}$ as well as the additional points generically so that there none are collinear with previously constructed points. Then, one can check that the cross-ratio of the solid points on the central line is the product of the cross-ratios on the other two lines. Set-theoretically, this claim follows from the fact 
that projections between parallel lines preserve ratios of distances, and so measuring from the leftmost point of the $x_{i}$ line, the top projection ensures that the ratio between the distances to the empty circle and the rightmost circle is $x_{j}$. Likewise, the lower projection ensures that the ratio of the distances to the center solid circle and the empty circle is $x_{k}$ and so $x_{i}=x_{j} x_{k}$ appears as the product of the ratios. If $j$ equals $k$, the diagram may be altered by moving the corresponding lines so that they coincide. In either case, the construction uses 6 additional points.

Second, the addition of variables $x_{i}=x_{j}+x_{k}$ can be constructed as in Fig. 5 . As in the case of multiplication, the motivation for this construction can be understood from the fact that projections scale distances. In particular, the top projection means that the empty circle and the outer points on the $x_{i}$ line encode the variable $x_{j}$, and since the two lower projections are both from points on the same horizontal line, the ratio of the distance between the inner points on the $x_{i}$ line to the distance between the outer points equals the value of $x_{k}$. Therefore, the intervals on either side of the empty circle encode the values of $x_{j}$ and $x_{k}$ and their concatenation computes $x_{i}=x_{j}+x_{k}$.

In the configuration from Fig. 5 , there will be an additional coincidence if $x_{j}=x_{k}$ in that the empty circle on the $x_{i}$ line, the middle point on the $x_{k}$ line and a point on the bottom line will be collinear. However, since $x_{j}+x_{k}, x_{j}$, and $x_{k}$ are all monic polynomials in the variable $t, x_{j}-x_{k}$ is also monic in $t$ and so a sufficiently generic choice of $t$ will ensure that $x_{j}-x_{k}$ is non-zero. Similarly if $x_{j}=-1$, then the empty point on the $x_{i}$ line, the rightmost point on the $x_{k}$ line and a marked point on the bottom line will be
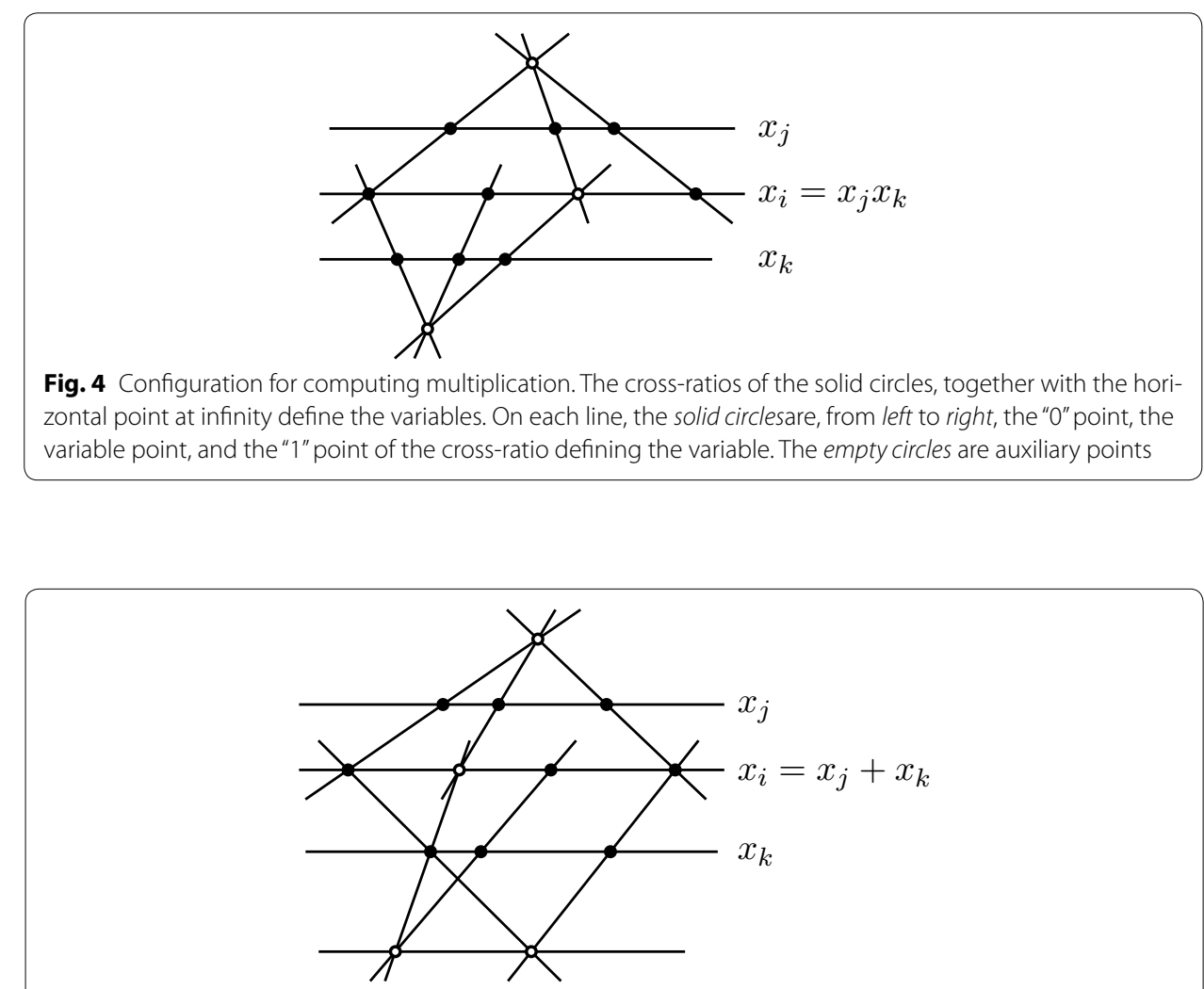

Fig. 5 Configuration for computing addition. The solid and empty circles represent the variables and auxiliary points, respectively, as in Fig. 4 
collinear, but this can again be avoided by adjusting $t$. For the addition operation, we have used 7 additional points.

Third, for incrementing a single variable, $x_{i}=x_{j}+1$, we specialize the configuration in Fig. 5 so that $x_{k}=1$, giving Fig. 6 . The line labeled with 1 can be chosen once and used in common for all increment operations, since it functions as a representative of the constant 1 . We have used 7 additional points for each increment operation, together with 2 points common to all such operations.

At this point, the realization space still surjects onto $\operatorname{Spec} S_{n}$, and so we still need to impose the equalities and inequalities. Each inequality $x_{i} \neq x_{j}$ can be imposed using the diagram in Fig. 7, which works by projecting the two variable points to the same line and getting different points. By replacing the projections of the two variables to the central line with the same point, we can use a similar figure to assert equality $x_{i}=x_{j}$. These use 6 and 5 additional points, respectively.

To summarize, we have agglomerated the configurations in Figs. 4, 5, 6, and 7 to give a matroid whose realization space projects to $\operatorname{Spec} R \subset \operatorname{Spec} S_{n}$. The realization of this matroid is determined by the values of the $y_{i}$, together with a number of parameters, such as the height of the horizontal lines, which are allowed to be generic, and thus the realization space is an open subset of $\mathbb{A}^{N} \times \operatorname{Spec} R$.

To show that the projection to $\operatorname{Spec} R$ is surjective, we take any point of $\operatorname{Spec} R$, which we can assume to be defined over an infinite field. Our construction of the realization required us to avoid certain coincidences, such as any $x_{i}$ being 0 or 1 or an equality

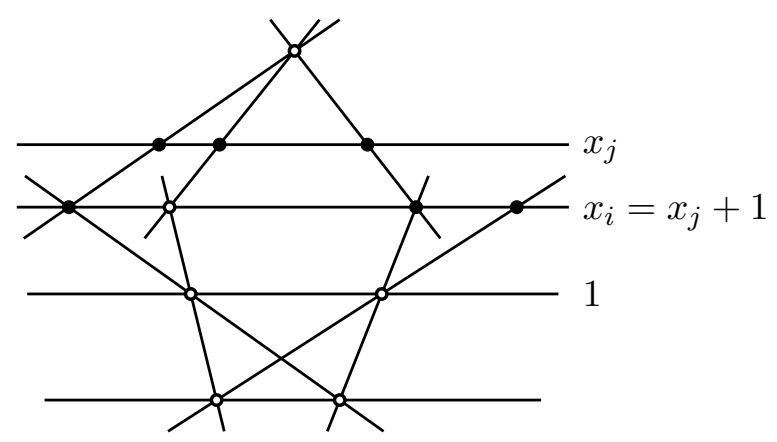

Fig. 6 Configuration for incrementing. The solid and empty circles represent the variables and the auxiliary points, respectively, as in Fig. 4 , with the exception that, on the $x_{i}$ line, the variable point is the rightmost solid point (since $\left.x_{i}>1\right)$

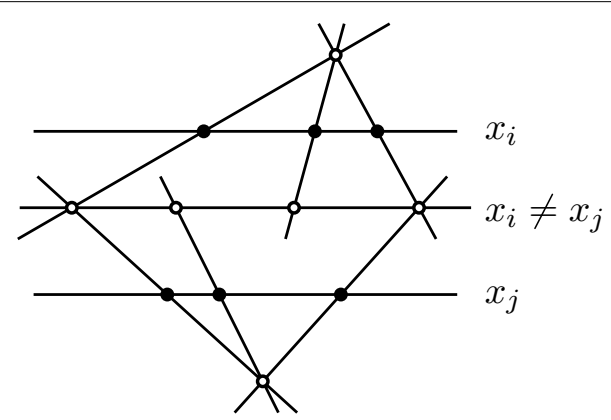

Fig. 7 Configuration for imposing inequality. The solid and empty circles represent the variables and auxiliary points, respectively, as in Fig. 4 
$x_{j}=x_{k}$ in any addition step. Each such coincidence only occurs for a finite number of possible values for $t$ we choose $t$ outside the union of all coincidences, and we can construct a realization of the matroid.

Finally, we justify the quantitative parts of the theorem statement. The number of elements of $M$ is computed by summing the number of elements for each of the building blocks together with 1 element for the common point on the horizontal lines, 3 elements for the variable $x_{0}=t$, and 2 elements for the horizontal line representing 1 in Fig. 6 . For the computation of $N$, we can assume that the coordinates on $\mathbb{P}^{2}$ are such that the common point of the horizontal lines is (1:0:0), the points representing 1 are (0:0:1) and (1:0:1), and the " 0 " and " 1 " points of the $x_{0}=t$ line are $(0: 1: 0)$ and $(0: 1: 1)$, respectively. These fix the automorphisms of $\mathbb{P}^{2}$. Then, one can check that each additional free variable and each of the building blocks adds 3 additional generic parameters. Finally, the value of $t$ is one more free parameter, which gives the expression for $N$.

Proof of Theorem 1.5 By Theorems 3.4, 3.5, and 5.3, it will be enough to construct sufficiently parsimonious elementary monic representations of the algebras $\mathbb{Z} / p$ and $\mathbb{Z}\left[p^{-1}\right]$ and thus matroids $M$ and $M^{\prime}$, respectively, representing these equations. Let $\ell$ be the largest integer less than $\sqrt{p}$. The elementary monic representations for both $M$ and $M^{\prime}$ use the equations shown in Fig. 8. Then, $\mathbb{Z} / p$ can be represented by adding an equality between $x_{\ell+5}$ and $x_{\ell+p-\ell^{2}+8}$ and $\mathbb{Z}\left[p^{-1}\right]$ can be represented by an inequality between the same pair of variables.

$$
\begin{aligned}
x_{0}= & t \\
x_{1}= & x_{0} x_{0}=t^{2} \\
x_{2}= & x_{0} x_{1}=t^{3} \\
x_{3}= & x_{0}+1=t+1 \\
& \vdots \\
x_{\ell+2}= & x_{\ell+1}+1=t+\ell \\
x_{\ell+3}= & x_{1}+x_{\ell+1}=t^{2}+t+\ell \\
x_{\ell+4}= & x_{\ell+3}+x_{\ell+1}=t^{2}+2 t+2 \ell \\
x_{\ell+5}= & x_{0} x_{\ell+4}=t^{3}+2 t^{2}+2 \ell t \\
x_{\ell+6}= & x_{\ell+2} x_{\ell+2}=t^{2}+2 \ell t+\ell^{2} \\
x_{\ell+7}= & x_{\ell+6}+1=t^{2}+2 \ell t+\ell^{2}+1 \\
& \vdots \\
x_{\ell+p-\ell^{2}+6}= & x_{\ell+p-\ell^{2}+5}+1=t^{2}+2 \ell t+p \\
x_{\ell+p-\ell^{2}+7}= & x_{2}+x_{\ell+p-\ell^{2}+6}=t^{3}+t^{2}+2 \ell t+p \\
x_{\ell+p-\ell^{2}+8}= & x_{1}+x_{\ell+p-\ell^{2}+7}=t^{3}+2 t^{2}+2 \ell t+p
\end{aligned}
$$

Fig. 8 System of equations used in the elementary monic representations of $\mathbb{Z} / p$ and $\mathbb{Z}\left[p^{-1}\right]$, where $p$ is a prime and $\ell$ denotes the largest integer less than $\sqrt{p}$ 
In either case, this representation uses no free variables, $\ell+p-\ell^{2}$ increments, 4 additions, and 4 multiplications. Thus, by Theorem 5.3, $M$ and $M^{\prime}$ have $7\left(\ell+p-\ell^{2}\right)+64$ and $7\left(\ell+p-\ell^{2}\right)+63$ elements, respectively. We will bound the former since it is larger. We first rewrite the number of elements as

$$
7\left(\ell+p-\ell^{2}\right)+64=p-\ell^{2}+7 \ell+6\left(p-\ell^{2}\right)+64
$$

To show that (4) is smaller than $p$, we note that since $p \geq 443$, then $\ell \geq 21$. We now have two cases. First, if $\ell=21$, then the largest prime number less than $22^{2}$ is 479 , so $p-n^{2} \leq 38$. Using this, we can bound (4) as

$$
p-21^{2}+7 \times 21+6(38)+64=p-2<p
$$

On the other hand, if $\ell \geq 22$, then the choice of $\ell$ means that $p<(\ell+1)^{2}$, so $p-\ell^{2} \leq 2 \ell$. Therefore, we can bound (4) as follows:

$$
\begin{aligned}
p-\ell^{2}+7 \ell+6(2 \ell)+64 & =p-\ell^{2}+19 \ell+64 \\
& =p-(\ell-19) \ell+64 \\
& \leq p-2<p
\end{aligned}
$$

Thus, the number of elements of $M$ and $M^{\prime}$ is less than $p$.

We take the graphs $\Gamma$ and $\Gamma^{\prime}$ and the divisors $D$ and $D^{\prime}$ for the theorem statement to be the matroid divisors of $M$ and $M^{\prime}$, respectively. Since $M$ and $M^{\prime}$ have fewer than $p$ elements, $D$ and $D^{\prime}$ have degree less than $p$. Moreover, since $k$ is an infinite field, by Theorems 3.4 and 3.5, $\Gamma$ and $D$ lift over $k[[t]]$ if and only if $M$ is representable over $k$, which means that the characteristic of $k$ equals $p$. Similarly, $\Gamma^{\prime}$ and $D^{\prime}$ lift if and only if the characteristic of $k$ is not $p$.

Remark 5.4 The threshold for $p$ in Theorem 1.5 is not optimal. For example, using a different construction when $p$ is closer to a larger square number than to a smaller square, it is possible to reduce the bound to 331 .

\section{Acknowledgements}

I would like to thank Spencer Backman, Melody Chan, Alex Fink, Eric Katz, Yoav Len, Diane Maclagan, Sam Payne, Kristin Shaw, and Ravi Vakil for helpful comments on this project. The project was begun while the author was supported by NSF Grant DMS-1103856L.

\section{Competing interests}

The author declares that he has no competing interests.

Received: 25 February 2015 Accepted: 11 September 2015

Published online: 10 November 2015

\section{References}

1. Amini, O., Baker, M.: Linear series on metrized complexes of algebraic curves. Math. Ann. 362(1), 55-106 (2015).arXiv:1204.3508

2. Amini, O., Caporaso, L.: Riemann-Roch theory for weighted graphs and tropical curves. Adv. Math. 240, 1-23 (2013).arXiv:1112.5134

3. Amini, O., Baker, M., Brugallé, E., Rabinoff, J.: Lifting harmonic morphisms II: Tropical curves and metrized complexes. Algebra Number Theory 9(2), 267-315 (2015).arXiv:1404.3390

4. Amini, O., Baker, M., Brugallé, E., Rabinoff, J.: Lifting harmonic morphisms I: metrized complexes and Berkovich skeleta. Res. Math. Sci. 2(7) (2015). arXiv:1303.4812 
5. Ardila, F., Klivans, C.: The Bergman complex of a matroid and phylogenetic trees. J. Combin. Theory Ser. B 96(1), 38-49 (2006). arXiv:math/0311370

6. Baker, M.: Specialization of linear systems from curves to graphs. Algebra Number Theory 2(6), 613-653 (2008). arXiv:math/0701075 (With an appendix by Brian Conrad)

7. Baker, M., Norine, S.: Riemann-Roch and Abel-Jacobi theory on a finite graph. Adv. Math. 215, 766-788 (2007). arXiv:math/0608360

8. Baker, M., Norine, S.: Harmonic morphisms and hyperelliptic graphs. Int. Math. Res. Not. 15, 2914-2955 (2009).arXiv:0707.1309

9. Caporaso, L.: Rank of divisors on graphs: an algebro-geometric analysis. In: A Celebration of Algebraic Geometry. Clay Math. Proc., vol. 18, pp. 45-64. Amer. Math. Soc., Providence (2013). arXiv:1204.3487

10. Cartwright, D., Jensen, D., Payne, S.: Lifting divisors on a generic chain of loops. Canad. Math. Bull. 58, 250-262 (2015)

11. Develin, M., Santos, F., Sturmfels, B.: On the rank of a tropical matrix. In: Combinatorial and Computational Geometry. Math. Sci. Res. Inst. Publ., vol. 52, pp. 213-242. Cambridge University Press, Cambridge (2005). arXiv:math/0312114

12. Dhar, D.: Self-organized critical state of the sandpile automaton models. Phys. Rev. Lett. 64, 1613-1616 (1990)

13. Eisenbud, D., Harris, J.: Limit linear series: basic theory. Invent. math. 85, 337-371 (1986)

14. Jensen, D.: The locus of Brill-Noether general graphs is not dense. preprint, arXiv:1405.6338 (2014)

15. Katz, E.: Matroid theory for algebraic geometers. Simons Symposium volume, to appear (2014). arXiv:1409.3503

16. Katz, E., Payne, S.: Realization spaces for tropical fans. In: Combinatorial Aspects of Commutative Algebra and Algebraic Geometry, vol. 6, pp. 73-88. Springer, Berlin (2011). Abel Symp. arXiv:0909.4582

17. Lafforgue, L.: Chirurgie des Grassmanniennes. CRM Monograph Series, vol. 19. American Mathematical Society, Providence (2003)

18. Lee, S.H., Vakil, R.: Mnëv-Sturmfels universality for schemes. In: A Celebration of Algebraic Geometry. Clay Math. Proc., vol. 18, pp. 457-468. Amer. Math. Soc., Providence (2013). arXiv:1202.3934

19. Len, Y.: A note on algebraic rank, matroids, and metrized complexes. preprint, arXiv:1410.8156 (2014)

20. Mnëv, N.E.: The universality theorems on the classification problem of configuration varieties and convex polytopes varieties. Lect. Notes Math. vol. 1346, pp. 527-543. Springer, Berlin (1988)

21. Oxley, J.: Matroid Theory. Oxford graduate texts in mathematics, vol. 21. Oxford University Press, Oxford (1992)

\section{Submit your manuscript to a SpringerOpen ${ }^{\circ}$ journal and benefit from:}

- Convenient online submission

- Rigorous peer review

- Immediate publication on acceptance

- Open access: articles freely available online

- High visibility within the field

- Retaining the copyright to your article

Submit your next manuscript at $\boldsymbol{\nabla}$ springeropen.com 